\title{
SET RECONSTRUCTION BY VORONOI CELLS
}

\author{
M. REITZNER, ${ }^{*}$ Universität Osnabrück \\ E. SPODAREV, ${ }^{* *}$ Universität Ulm \\ D. ZAPOROZHETS, ${ }^{* * *}$ V. A. Steklov Mathematical Institute
}

\begin{abstract}
For a Borel set $A$ and a homogeneous Poisson point process $\eta$ in $\mathbb{R}^{d}$ of intensity $\lambda>0$, define the Poisson-Voronoi approximation $A_{\eta}$ of $A$ as a union of all Voronoi cells with nuclei from $\eta$ lying in $A$. If $A$ has a finite volume and perimeter, we find an exact asymptotic of $\operatorname{EVol}\left(A \Delta A_{\eta}\right)$ as $\lambda \rightarrow \infty$, where $\operatorname{Vol}$ is the Lebesgue measure. Estimates for all moments of $\operatorname{Vol}\left(A_{\eta}\right)$ and $\operatorname{Vol}\left(A \Delta A_{\eta}\right)$ together with their asymptotics for large $\lambda$ are obtained as well.
\end{abstract}

Keywords: Poisson point process; Poisson-Voronoi cell; Poisson-Voronoi tessellation; perimeter

2010 Mathematics Subject Classification: Primary 60D05

Secondary 60G55; 52A22; 60C05

\section{Introduction}

Let $A$ be a Borel set in $\mathbb{R}^{d}$, and let $\eta$ be a Poisson point process in $\mathbb{R}^{d}$. Assume that we observe $\eta$ and that the only information about $A$ at our disposal is which points of $\eta$ lie in $A$, i.e. we have the partition of the process $\eta$ into $\eta \cap A$ and $\eta \backslash A$. We try to reconstruct the set $A$ just by the information contained in these two point sets. To this end, we approximate $A$ by the set $A_{\eta}$ of all points in $\mathbb{R}^{d}$ which are closer to $\eta \cap A$ than to $\eta \backslash A$.

More formally, let $\eta$ be a homogeneous Poisson point process of intensity $\lambda>0$, and denote by $v_{\eta}(x)=\left\{z \in \mathbb{R}^{d}:\|z-x\| \leq\|z-y\|\right.$ for all $\left.y \in \eta\right\}$ the Voronoi cell generated by $\eta$ with nucleus $x \in \eta$. Then the set $A_{\eta}$ is just the union of the Poisson-Voronoi cells with nuclei lying in $A$, i.e.

$$
A_{\eta}=\bigcup_{x \in \eta \cap A} v_{\eta}(x) .
$$

We call this set the Poisson-Voronoi approximation of the set $A$. It was first introduced by Khmaladze and Toronjadze [8]. They proposed $A_{\eta}$ to be an estimator for $A$ when $\lambda$ is large (potential applications are listed in [7, Section 1]). They conjectured that, for an arbitrary

Received 3 January 2012; revision received 2 March 2012.

* Postal address: Institut für Mathematik, Universität Osnabrück, 49069 Osnabrück, Germany.

Email address: matthias.reitzner@uni-osnabrueck.de

** Postal address: Institut für Stochastik, Universität Ulm, 89069 Ulm, Germany.

Email address: evgeny.spodarev@uni-ulm.de

Partially supported by RFBR-DFG (09-0191331) and DFG (436 RUS 113/962/0-1 R) grants.

*** Postal address: St. Petersburg Department, V. A. Steklov Mathematical Institute, Fontanka 27, 191023

St. Petersburg, Russia. Email address: zap1979@gmail.com

Partially supported by RFBR (10-01-00242), NSh-4472.2010.1, RFBR-DFG (09-0191331), and DFG (436 RUS

113/962/0-1 R) grants. 
bounded Borel set $A \subset \mathbb{R}^{d}, d \geq 1$, it holds that

$$
\begin{gathered}
\operatorname{Vol}\left(A_{\eta}\right) \rightarrow \operatorname{Vol}(A) \quad \text { as } \lambda \rightarrow \infty, \\
\operatorname{Vol}\left(A \Delta A_{\eta}\right) \rightarrow 0 \quad \text { as } \lambda \rightarrow \infty,
\end{gathered}
$$

almost surely, where $\operatorname{Vol}(\cdot)$ stands for the Lebesgue measure (volume) and $\Delta$ is the operation of the symmetric difference of sets. This conjecture was proved in [8] for $d=1$. The case of general $d$ was treated by Einmahl and Khmaladze [5] with some technical assumption on the boundary of $A$, and then generalized by Penrose [11] to an arbitrary bounded Borel set $A$.

It can be easily shown (see Section 3 for details) that, for any Borel set $A$, it holds that

$$
\operatorname{EVol}\left(A_{\eta}\right)=\operatorname{Vol}(A)
$$

Thus, $\operatorname{Vol}\left(A_{\eta}\right)$ is an unbiased estimator for the volume of $A$. In this paper we also consider the $n$th moment of $\operatorname{Vol}\left(A_{\eta}\right)$ and approximate it by the $n$th degree of the volume of the original set $\mathrm{Vol}^{n}(A)$ asymptotically as $\lambda \rightarrow \infty$ (Theorem 2). For the case when $n=2$ and $A$ is a convex compact, similar estimates were obtained in [7].

It might be suggested from (1) that

$$
\mathrm{E} \operatorname{Vol}\left(A \Delta A_{\eta}\right) \rightarrow 0 \quad \text { as } \lambda \rightarrow \infty
$$

although it is not a direct corollary. The more interesting problem is to find an exact asymptotic of $\mathrm{E} \operatorname{Vol}\left(A \Delta A_{\eta}\right)$. This problem was initially considered by Heveling and Reitzner [7]. They proved that, for any compact convex set $A$ with $(d-1)$-dimensional Hausdorff measure $\mathscr{H}_{d-1}(\partial A)$ of the boundary $\partial A$ of $A$, it holds that

$$
\mathrm{E} \operatorname{Vol}\left(A \Delta A_{\eta}\right)=c_{d} \mathscr{H}_{d-1}(\partial A) \lambda^{-1 / d}\left(1+O\left(\lambda^{-1 / d}\right)\right) \quad \text { as } \lambda \rightarrow \infty
$$

where the constant $c_{d}$ is independent of $\lambda$ and $A$ was calculated by them in an explicit form (see Section 2 for details). Here we obtain a similar asymptotic formula (Theorem 1) for a much wider class of sets. Namely, we consider Borel sets with finite volume $\operatorname{Vol}(A)$ and perimeter $\operatorname{Per}(A)$ (see Section 3 for the precise definition). Our methods are completely different from those of Heveling and Reitzner. The key observations are the connection between the Poisson-Voronoi approximation and the covariogram of $A$, and the connection between the covariogram and the perimeter of a set recently established by Galerne [6]. As a by-product of our calculations, we prove that (2) holds for any Borel set $A$ with finite volume (Corollary 3 ).

We also consider higher moments of $\operatorname{Vol}\left(A \Delta A_{\eta}\right)$. For an arbitrary Borel set $A$, we approximate $\mathrm{E} \mathrm{Vol}^{n}\left(A \Delta A_{\eta}\right)$ by the $n$th degree of $\mathrm{E} \operatorname{Vol}\left(A \Delta A_{\eta}\right)$ asymptotically as $\lambda \rightarrow \infty$ (Theorem 3$)$. Thus, assuming that $\operatorname{Vol}(A), \operatorname{Per}(A)<\infty$ and using the asymptotic for $\operatorname{E} \operatorname{Vol}\left(A \Delta A_{\eta}\right)$ from Theorem 1, we obtain the asymptotic for $\mathrm{E} \mathrm{Vol}^{n}\left(A \Delta A_{\eta}\right)$ (Corollary 1).

The paper is organized as follows. Our main results are stated in Section 2. In Section 3 we introduce the necessary background and notation, in particular the perimeter and the covariogram of a set $A$. Proofs are given in Section 4.

\section{Main results}

Our first result yields the asymptotic of the average volume of $A \Delta A_{\eta}$ with increasing intensity $\lambda$. To formulate it, we need to define the notion of a perimeter of a Borel set. The definition is somewhat technical, so we postpone it till Section 3. If $A$ is a compact 
set with Lipschitz boundary (e.g. a convex body), then $\operatorname{Per}(A)$ equals the $(d-1)$-dimensional Hausdorff measure $\mathscr{H}_{d-1}(\partial A)$ of the boundary $\partial A$ of $A$. In the general case it holds that $\operatorname{Per}(A) \leq \mathscr{H}_{d-1}(\partial A)$ (see, e.g. [2, Proposition 3.62]). Therefore, $\operatorname{Per}(A)$ could be replaced by $\mathscr{H}_{d-1}(\partial A)$ in the assumptions of the theorem.

Theorem 1. If $A \subset \mathbb{R}^{d}$ is a Borel set with $\operatorname{Vol}(A)<\infty$ and $\operatorname{Per}(A)<\infty$, then

$$
\operatorname{EVol}\left(A \Delta A_{\eta}\right)=c_{d} \operatorname{Per}(A) \lambda^{-1 / d}(1+o(1)) \text { as } \lambda \rightarrow \infty,
$$

where $c_{d}=2 d^{-2} \Gamma(1 / d) \kappa_{d-1} \kappa_{d}^{-1-1 / d}$ and $\kappa_{n}$ is the volume of the unit n-dimensional ball.

The probabilistic intuition behind this asymptotic is the following. The set difference $A \Delta A_{\eta}$ is formed out of the Poisson-Voronoi cells with nuclei lying almost on $\partial A$. Thus, its volume behaves asymptotically like the volume of a small tube neighbourhood $B(\partial A)$ of the boundary $\partial A$. The size of the boundary is given by the perimeter of $A$. Since the volume of a typical Poisson-Voronoi cell is $\lambda^{-1}$, the width of this tube neighbourhood should have order $\lambda^{-1 / d}$, and so the volume of the tube neighbourhood has the order $\operatorname{Per}(A) \lambda^{-1 / d}$.

In the following, when we say that some inequality holds asymptotically as $\lambda \rightarrow \infty$, we mean that it holds for sufficiently large $\lambda \geq \lambda_{0}$. The choice of $\lambda_{0}$ might depend on $A$. Thus, all estimates are not uniform with respect to $A$ (including those of Theorem 1).

Theorem 2. If $A \subset \mathbb{R}^{d}$ is a Borel set with $\operatorname{Vol}(A)<\infty$ then

$$
\left|\mathrm{EVol}^{n}\left(A_{\eta}\right)-\operatorname{Vol}^{n}(A)\right| \leq C_{n, d} \operatorname{Vol}^{n-1}(A) \lambda^{-1} \text { as } \lambda \rightarrow \infty,
$$

where $C_{n, d}$ is some constant independent of $\lambda$ and $A$.

Remark 1. In fact, we show that the following nonasymptotic inequality holds: for any $\lambda>0$,

$$
\left|\mathrm{EVol}^{n}\left(A_{\eta}\right)-\operatorname{Vol}^{n}(A)\right| \leq C_{n, d} \sum_{k=1}^{n-1} \operatorname{Vol}^{n-k}(A) \lambda^{-k}
$$

Theorem 3. If $A \subset \mathbb{R}^{d}$ is a Borel set with $\operatorname{Vol}(A)<\infty$ and $\operatorname{Per}(A)<\infty$, then

$$
\left|\mathrm{EVol}^{n}\left(A \Delta A_{\eta}\right)-\left(\mathrm{EVol}\left(A \Delta A_{\eta}\right)\right)^{n}\right| \leq C_{n, d}^{\prime} \operatorname{Per}(A)^{n-1} \lambda^{-1-(n-1) / d} \text { as } \lambda \rightarrow \infty,
$$

where $C_{n, d}^{\prime}$ is some constant independent of $\lambda$ and $A$.

Remark 2. We conjecture that the following limit theorems can be proven by the method of moments (see, e.g. [4, Theorems 30.1, 30.2]):

$$
\begin{gathered}
\lambda^{1 / 2(1+1 / d)}\left(\operatorname{Vol}\left(A_{\eta}\right)-\operatorname{Vol}(A)\right) \rightarrow N\left(0, \sigma_{1} \operatorname{Per}(A)\right), \\
\lambda^{1 / 2(1+1 / d)}\left(\operatorname{Vol}\left(A \Delta A_{\eta}\right)-c_{d} \operatorname{Per}(A) \lambda^{-1 / d}\right) \rightarrow N\left(0, \sigma_{2} \operatorname{Per}(A)\right)
\end{gathered}
$$

in distribution as $\lambda \rightarrow \infty, \sigma_{1}, \sigma_{2}>0$.

Recently, (4) was proved by Schulte [13] for convex sets $A$ using a central limit theorem for Wiener-Itô chaos expansions. In his Remark 4 Schulte points out that the result can be extended to all sets where the volume of a small tube neighbourhood $B(\partial A)$ of $\partial A$ can be bounded in a nice way. Yet the general conjecture seems to be open. 
Corollary 1. If $A \subset \mathbb{R}^{d}$ is a Borel set with $\operatorname{Vol}(A)<\infty$ and $\operatorname{Per}(A)<\infty$, then

$$
\mathrm{EVol}^{n}\left(A \Delta A_{\eta}\right)=\left(\mathrm{EVol}\left(A \Delta A_{\eta}\right)\right)^{n}\left(1+O\left(\lambda^{-1+1 / d}\right)\right) \text { as } \lambda \rightarrow \infty,
$$

and, for $d \geq 2$,

$$
\mathrm{E} \mathrm{Vol}^{n}\left(A \Delta A_{\eta}\right)=\left(c_{d} \operatorname{Per}(A)\right)^{n} \lambda^{-n / d}(1+o(1)) \text { as } \lambda \rightarrow \infty .
$$

The asymptotic order of the variance of $A_{\eta}$ and $A \Delta A_{\eta}$ as $\lambda \rightarrow \infty$ was first studied in [7] for convex sets $A$. We extend these results to arbitrary Borel sets.

Corollary 2. If $A \subset \mathbb{R}^{d}$ is a Borel set with $\operatorname{Vol}(A)<\infty$ and $\operatorname{Per}(A)<\infty$, then

$$
\operatorname{var} \operatorname{Vol}\left(A_{\eta}\right) \leq C_{d} \operatorname{Per}(A) \lambda^{-1-1 / d} \text { as } \lambda \rightarrow \infty
$$

and

$$
\operatorname{var} \operatorname{Vol}\left(A \Delta A_{\eta}\right) \leq C_{d} \operatorname{Per}(A) \lambda^{-1-1 / d} \text { as } \lambda \rightarrow \infty,
$$

where $C_{d}$ is some constant independent of $\lambda$ and $A$.

The second inequality follows immediately from Theorem 3. The first inequality will be proved in Section 4.2.

The probabilistic heuristic explaining the asymptotic behaviour of the variances is the following. The symmetric difference $A \Delta A_{\eta}$ consists of parts $\tilde{v}_{\eta}(x)$ of almost independent Poisson-Voronoi cells $v_{\eta}(x)$. Their nuclei $x \in B(\partial A)$ are asymptotically contained in a small tube neighbourhood $B(\partial A)$ of $\partial A$ already mentioned in the heuristic intuition after Theorem 1. We use the formula for the variance of the compound Poisson distribution,

$$
\operatorname{var} \operatorname{Vol}\left(A \Delta A_{\eta}\right)=\operatorname{var}\left(\sum_{x \in \eta \cap B(\partial A)} \operatorname{Vol}\left(\tilde{v}_{\eta}(x)\right)\right) \approx \operatorname{var}\left(\sum_{i=1}^{N} Y_{i}\right),
$$

where the random variables $Y_{i} \stackrel{\mathrm{D}}{=} \operatorname{Vol}\left(\tilde{v}_{\eta}(x)\right)$ are independent and identically distributed and

$$
N \stackrel{\mathrm{D}}{=} \operatorname{card}(\eta \cap B(\partial A)) \sim \operatorname{Pois}(\lambda \operatorname{Vol}(B(\partial A)))
$$

is independent of $Y_{i}$. Here ' $\stackrel{\text { D }}{=}$ denotes equality in distribution and $\operatorname{card}(B)$ is the cardinality of a set $B$. Then

$$
\begin{aligned}
\operatorname{var}\left(\sum_{i=1}^{N} Y_{i}\right) & =\mathrm{E} N \operatorname{var} Y_{1}+\operatorname{var} N\left(\mathrm{E} Y_{1}\right)^{2} \\
& =\lambda \operatorname{Vol}(B(\partial A)) \mathrm{E} Y_{1}^{2} \\
& \leq \lambda \operatorname{Vol}(B(\partial A))\left(\mathrm{E} \operatorname{Vol}\left(v_{\eta}(x)\right)\right)^{2} \\
& =O\left(\lambda \operatorname{Per}(A) \lambda^{-1 / d} \lambda^{-2}\right) \\
& =\operatorname{Per}(A) O\left(\lambda^{-1-1 / d}\right)
\end{aligned}
$$

since $\tilde{v}_{\eta}(x) \subset v_{\eta}(x)$ for any $x$, the second moment of the volume of a typical Poisson-Voronoi cell is of order $\lambda^{-2}$, and the volume of $B(\partial A)$ is of order $\operatorname{Per}(A) \lambda^{-1 / d}$. 
The results of Corollary 2 can also be obtained by using the Poincaré inequality which gives an upper bound on the variance of a functional of a Poisson point process. Let $\mathcal{N}$ be the set of all locally finite configurations on $\mathbb{R}^{d}$. Consider a nonnegative measurable function $F: \mathcal{N} \rightarrow \mathbb{R}$. If $F^{2}(\eta)<\infty$ then

$$
\operatorname{var} F(\eta) \leq \lambda \mathrm{E} \int_{\mathbb{R}^{d}}(F(\eta \cup\{y\})-F(\eta))^{2} \mathrm{~d} y,
$$

where we have added a point $y$ to the Poisson point process $\eta$. Putting $F(\eta)=\operatorname{Vol}\left(A_{\eta}\right)$ in (5), we obtain

$$
\operatorname{var} \operatorname{Vol}\left(A_{\eta}\right) \leq \lambda \int_{\mathbb{R}^{d}} \mathrm{E}\left(\operatorname{Vol}\left(A_{\eta \cup\{y\}}\right)-\operatorname{Vol}\left(A_{\eta}\right)\right)^{2} \mathrm{~d} y,
$$

where the right-hand side can be estimated from above to obtain the upper bound in Corollary 2 . The reasoning for the symmetric difference $A \Delta A_{\eta}$ is similar.

In full generality, inequality (5) was proved by $\mathrm{Wu}$ [15]. As was shown by Last and Penrose [9, Theorem 1.2], it is a consequence of an even more general inequality following from the Fock space representation of Poisson point processes.

\section{Preliminaries}

For basic facts from integral geometry, stochastic geometry, and Voronoi tessellations which are not explained in the following, we refer the reader to [10], [12], and [14].

Define the perimeter of a Borel set $A$ as

$$
\operatorname{Per}(A)=\sup \left\{\int_{A} \operatorname{div} \varphi(x) \mathrm{d} x: \varphi \in \mathcal{C}_{c}^{1}\left(\mathbb{R}^{d}\right),\|\varphi\|_{\infty} \leq 1\right\},
$$

cf. [2], where

$$
\operatorname{div} \varphi(x)=\sum_{i=1}^{d} \frac{\partial \varphi_{i}}{\partial x_{i}} \quad \text { and } \quad\|\varphi\|_{\infty}=\max _{i=1, \ldots, d} \sup _{x \in \mathbb{R}^{d}}\left|\varphi_{i}(x)\right|
$$

for $\varphi=\left(\varphi_{1}, \ldots, \varphi_{d}\right)$. The class $\mathcal{C}_{c}^{1}\left(\mathbb{R}^{d}\right)$ consists of all continuously differentiable vectorvalued functions from $\mathbb{R}^{d}$ to $\mathbb{R}^{d}$ with compact support. It is well known that if $A$ is a compact set with Lipschitz boundary (e.g. a convex body), then $\operatorname{Per}(A)$ equals the $(d-1)$-dimensional Hausdorff measure $\mathscr{H}_{d-1}(\partial A)$ of the boundary $\partial A$ of $A$. Therefore, in most applications, $\operatorname{Per}(A)$ could be replaced by $\mathscr{H}_{d-1}(\partial A)$. In the general case,

$$
\operatorname{Per}(A) \leq \mathscr{H}_{d-1}(\partial A)
$$

(see, e.g. [2, Proposition 3.62]). More detailed information about the perimeter of a set and its connection to the Hausdorff measure of the boundary is given in [1] where, e.g. it is proved [1, Theorem 3] that the perimeter equals the Hausdorff measure of the essential boundary of the set if the perimeter is finite.

Let $A$ be a Borel set with finite volume. Then

$$
g_{A}(x)=\operatorname{Vol}((A+x) \cap A), \quad x \in \mathbb{R}^{d},
$$

is a covariogram of $A$. For the history on the covariogram problem, see the references in [6] and also the recent breakthrough by Averkov and Bianchi [3]. 
In the proof of Theorem 1 we use the result obtained by Galerne [6, Theorem 14]. The following assertions are equivalent:

(a) $\operatorname{Per}(A)<\infty$;

(b) there exists a finite limit

$$
\lim _{r \rightarrow+0} \frac{g_{A}(r u)-g_{A}(0)}{r}=\frac{\partial g_{A}}{\partial u}(0)
$$

for all $u \in \mathbb{S}^{d-1}$

(c) $g_{A}$ is Lipschitz.

In addition, the Lipschitz constant of $g_{A}$ satisfies

$$
\operatorname{Lip}\left(g_{A}\right) \leq \frac{1}{2} \operatorname{Per}(A)
$$

and it holds that

$$
\int_{\mathbb{S}^{d-1}} \frac{\partial g_{A}}{\partial u}(0) \mathscr{H}_{d-1}(\mathrm{~d} u)=-\kappa_{d-1} \operatorname{Per}(A) .
$$

Another tool we need is the refined Campbell-Mecke formula for stationary point processes (cf., e.g. [14]). Using Slivnyak's theorem, we give its particular case for the Poisson point process.

As above, let $\eta$ be a homogeneous Poisson point process of intensity $\lambda>0$, and let $\mathcal{N}$ be the set of all locally finite point configurations on $\mathbb{R}^{d}$. Consider a nonnegative measurable function $f: \mathcal{N} \times\left(\mathbb{R}^{d}\right)^{m} \rightarrow \mathbb{R}$. Then

$$
\mathrm{E} \sum_{\left(y_{1}, \ldots, y_{m}\right) \in \eta_{\neq}^{m}} f\left(\eta, y_{1}, \ldots, y_{m}\right)=\lambda^{m} \int_{\left(\mathbb{R}^{d}\right)^{m}} \mathrm{E} f\left(\eta \cup \bar{y}_{m}, y_{1}, \ldots, y_{m}\right) \mathrm{d} y_{1} \cdots \mathrm{d} y_{m},
$$

where $\eta_{\neq}^{m}$ denotes the set of all $m$-tuples of pairwise distinct points from $\eta$, and $\eta \cup \bar{y}_{m}$ is the process $\eta$ with added point set $\bar{y}_{m}=\left\{y_{1}, \ldots, y_{m}\right\}$.

As a simple corollary we get two identities which are crucial for us in the sequel.

Proposition 1. If $A \subset \mathbb{R}^{d}$ is a Borel set with $\operatorname{Vol}(A)<\infty$ then

$$
\operatorname{EVol}\left(A_{\eta}\right)=\lambda \int_{\mathbb{R}^{d}} \int_{A} \mathrm{e}^{-\lambda \kappa_{d}\|y-x\|^{d}} \mathrm{~d} y \mathrm{~d} x=\operatorname{Vol}(A)
$$

and

$$
\operatorname{EVol}\left(A \Delta A_{\eta}\right)=2 \lambda \int_{\mathbb{R}^{d} \backslash A} \int_{A} \mathrm{e}^{-\lambda \kappa_{d}\|y-x\|^{d}} \mathrm{~d} y \mathrm{~d} x .
$$

Proof. By Fubini's theorem and the Slivnyak-Mecke formula (9), we have

$$
\begin{aligned}
\operatorname{EVol}\left(A_{\eta}\right) & =\mathrm{E} \int_{\mathbb{R}^{d}} \mathbf{1}\left(x \in A_{\eta}\right) \mathrm{d} x \\
& =\int_{\mathbb{R}^{d}} \mathrm{E} \sum_{y \in \eta \cap A} \mathbf{1}\left(x \in v_{\eta}(y)\right) \mathrm{d} x \\
& =\lambda \int_{\mathbb{R}^{d}} \int_{A} \mathrm{P}\left(x \in v_{\eta \cup\{y\}}(y)\right) \mathrm{d} y \mathrm{~d} x \\
& =\lambda \int_{\mathbb{R}^{d}} \int_{A} \mathrm{e}^{-\lambda \kappa_{d}\|x-y\|^{d}} \mathrm{~d} y \mathrm{~d} x .
\end{aligned}
$$


Similarly, we obtain

$$
\operatorname{EVol}\left(A \backslash A_{\eta}\right)=\lambda \int_{A} \int_{\mathbb{R}^{d} \backslash A} \mathrm{e}^{-\lambda \kappa_{d}\|x-y\|^{d}} \mathrm{~d} y \mathrm{~d} x
$$

and

$$
\operatorname{EVol}\left(A_{\eta} \backslash A\right)=\lambda \int_{\mathbb{R}^{d} \backslash A} \int_{A} \mathrm{e}^{-\lambda \kappa_{d}\|x-y\|^{d}} \mathrm{~d} y \mathrm{~d} x .
$$

By definition, $\operatorname{Vol}\left(A \Delta A_{\eta}\right)=\operatorname{Vol}\left(A \backslash A_{\eta}\right)+\operatorname{Vol}\left(A_{\eta} \backslash A\right)$, which completes the proof of (11). To prove the second part of (10), we have to apply Fubini's theorem and then use the formula

$$
\int_{\mathbb{R}^{d}} \mathrm{e}^{-c\|x-y\|^{d}} \mathrm{~d} x=\frac{\kappa_{d}}{c}, \quad c>0,
$$

which could be easily proved by introducing spherical coordinates.

Note that we have also proved that

$$
\mathrm{E} \operatorname{Vol}\left(A \backslash A_{\eta}\right)=\mathrm{EVol}\left(A_{\eta} \backslash A\right) .
$$

However, $\operatorname{Vol}\left(A \backslash A_{\eta}\right)$ and $\operatorname{Vol}\left(A_{\eta} \backslash A\right)$ are not equidistributed since the first random variable is bounded, and the second is not. As a direct corollary of identity (10), we obtain

$$
\operatorname{var} \operatorname{Vol}\left(A_{\eta}\right)=\mathrm{E}\left(\operatorname{Vol}\left(A \backslash A_{\eta}\right)-\operatorname{Vol}\left(A_{\eta} \backslash A\right)\right)^{2},
$$

which we shall use in the following.

\section{Proofs}

\subsection{Asymptotics of the mean volume of the symmetric difference}

In this section we give the proof of Theorem 1. The key step to prove it is the following relation between the Poisson-Voronoi approximation and the covariogram of a set $A$.

Lemma 1. Let $g_{A}(x)$ be the covariogram of a Borel set $A$ with $\operatorname{Vol}(A)<\infty$. Then

$$
\mathrm{E} \operatorname{Vol}\left(A \Delta A_{\eta}\right)=-2 \int_{0}^{\infty} r^{d-1} \mathrm{e}^{-\kappa_{d} r^{d}} \tilde{g}_{A}\left(\lambda^{-1 / d} r\right) \mathrm{d} r
$$

where

$$
\tilde{g}_{A}(r)=\int_{\mathbb{S}^{d-1}}\left(g_{A}(r u)-g_{A}(0)\right) \mathscr{H}_{d-1}(\mathrm{~d} u) .
$$

Proof. Replacing $y$ in (11) by $x-\lambda^{-1 / d} z$ we obtain

$$
\begin{aligned}
\operatorname{EVol}\left(A \Delta A_{\eta}\right) & =2 \lambda \int_{\mathbb{R}^{d}} \int_{\mathbb{R}^{d}} \mathrm{e}^{-\lambda \kappa_{d}\|y-x\|^{d}} \mathbf{1}\left(y \in A, x \in A^{\mathrm{c}}\right) \mathrm{d} y \mathrm{~d} x \\
& =2 \int_{\mathbb{R}^{d}} \mathrm{e}^{-\kappa_{d}\|z\|^{d}} \int_{\mathbb{R}^{d}} \mathbf{1}\left(x \in\left(A+\lambda^{-1 / d} z\right) \cap A^{\mathrm{c}}\right) \mathrm{d} z \mathrm{~d} x \\
& =2 \int_{\mathbb{R}^{d}} \mathrm{e}^{-\kappa_{d}\|z\|^{d}} \operatorname{Vol}\left(\left(A+\lambda^{-1 / d} z\right) \cap A^{\mathrm{c}}\right) \mathrm{d} z .
\end{aligned}
$$

By the definition of the covariogram,

$$
\operatorname{Vol}\left(\left(A+\lambda^{-1 / d} z\right) \cap A^{\mathrm{c}}\right)=g_{A}(0)-g_{A}\left(\lambda^{-1 / d} z\right) .
$$


We introduce spherical coordinates $z=r u$, where $r \in \mathbb{R}^{+}$and $u \in \mathbb{S}^{d-1}$. This yields

$$
\mathrm{EVol}\left(A \Delta A_{\eta}\right)=-2 \int_{0}^{\infty} r^{d-1} \mathrm{e}^{-\kappa_{d} r^{d}}\left[\int_{\mathbb{S}^{d-1}}\left(g_{A}\left(\lambda^{-1 / d} r u\right)-g_{A}(0)\right) \mathscr{H}_{d-1}(\mathrm{~d} u)\right] \mathrm{d} r
$$

Corollary 3. For any measurable A with $\operatorname{Vol}(A)<\infty$, it holds that

$$
\mathrm{E} \operatorname{Vol}\left(A \Delta A_{\eta}\right) \rightarrow 0 \quad \text { as } \lambda \rightarrow \infty
$$

Proof. The proof immediately follows from (14) and the continuity of the set covariogram.

Proof of Theorem 1. Using Lemma 1 and substituting $t$ for $\kappa_{d} r^{d}$, we obtain

$$
\operatorname{EVol}\left(A \Delta A_{\eta}\right)=-\frac{2}{d \kappa_{d}} \int_{0}^{\infty} \mathrm{e}^{-t} \tilde{g}_{A}\left(\left(\lambda \kappa_{d}\right)^{-1 / d} t^{1 / d}\right) \mathrm{d} t
$$

It follows from (7) and the definition of $\tilde{g}_{A}$ that

$$
\left|\tilde{g}_{A}(r)\right| \leq \frac{1}{2} \mathscr{H}_{d-1}\left(\mathbb{S}^{d-1}\right) \operatorname{Per}(A) r .
$$

Therefore, Lebesgue's dominated convergence theorem, (6), and (8) yield

$$
\begin{aligned}
\lim _{\lambda \rightarrow \infty} \operatorname{EVol}\left(A \Delta A_{\eta}\right) \lambda^{1 / d} & =-\frac{2}{d} \kappa_{d}^{-1-1 / d} \lim _{\lambda \rightarrow \infty} \int_{0}^{\infty} \mathrm{e}^{-t} t^{1 / d} \frac{\tilde{g}_{A}\left(\left(\lambda \kappa_{d}\right)^{-1 / d} t^{1 / d}\right)}{\left(\lambda \kappa_{d}\right)^{-1 / d} t^{1 / d}} \mathrm{~d} t \\
& =-\frac{2}{d} \kappa_{d}^{-1-1 / d} \int_{0}^{\infty} \mathrm{e}^{-t} t^{1 / d} \mathrm{~d} t \int_{\mathbb{S}^{d-1}} \frac{\partial g_{A}}{\partial u}(0) \mathscr{H}_{d-1}(\mathrm{~d} u) \\
& =\frac{2}{d} \kappa_{d-1} \kappa_{d}^{-1-1 / d} \operatorname{Per}(A) \int_{0}^{\infty} \mathrm{e}^{-t} t^{1 / d} \mathrm{~d} t \\
& =\frac{2}{d} \kappa_{d-1} \kappa_{d}^{-1-1 / d} \Gamma\left(1+\frac{1}{d}\right) \operatorname{Per}(A) .
\end{aligned}
$$

\subsection{Asymptotics of higher moments}

To prove Theorem 2 and Theorem 3, we need a number of lemmas. In this section, $C$ is always some constant independent of $\lambda$ and $A$. Our first statement is the following version of Hölder's inequality.

Lemma 2. For any events $A_{1}, \ldots, A_{m}$, it holds that

$$
\mathrm{P}\left(\bigcap_{r=1}^{m} A_{r}\right) \leq \prod_{r=1}^{m}\left(\mathrm{P}\left(A_{r}\right)\right)^{1 / m}
$$

Lemma 3. Let $x_{0}, y_{0} \in \mathbb{R}^{d}$. For any $\varepsilon>0$ and $m \in \mathbb{N}$, the following inequality holds:

$$
\begin{aligned}
\int_{\left(\mathbb{R}^{d}\right)^{m}} & \left(\mathrm{P}\left(x_{0}, x_{1}, \ldots, x_{m} \in v_{\eta \cup\left\{y_{0}\right\}}\left(y_{0}\right)\right)\right)^{\varepsilon} \mathrm{d} x_{1} \cdots \mathrm{d} x_{m} \\
\leq & \exp \left(-\frac{\varepsilon \lambda \kappa_{d}\left\|x_{0}-y_{0}\right\|^{d}}{m+1}\right)\left(\frac{m+1}{\varepsilon \lambda}\right)^{m} .
\end{aligned}
$$


Proof. By Lemma 2, we have

$$
\begin{aligned}
\int_{\left(\mathbb{R}^{d}\right)^{m}} & \left(\mathrm{P}\left(x_{0}, x_{1}, \ldots, x_{m} \in v_{\eta \cup\left\{y_{0}\right\}}\left(y_{0}\right)\right)\right)^{\varepsilon} \mathrm{d} x_{1} \cdots \mathrm{d} x_{m} \\
\leq & \left(\mathrm{P}\left(x_{0} \in v_{\eta \cup\left\{y_{0}\right\}}\left(y_{0}\right)\right)\right)^{\varepsilon /(m+1)} \int_{\left(\mathbb{R}^{d}\right)^{m}} \prod_{i=1}^{m}\left(\mathrm{P}\left(x_{i} \in v_{\eta \cup\left\{y_{0}\right\}}\left(y_{0}\right)\right)\right)^{\varepsilon /(m+1)} \mathrm{d} x_{1} \cdots \mathrm{d} x_{m} \\
= & \exp \left(\frac{-\varepsilon \lambda \kappa_{d}\left\|x_{0}-y_{0}\right\|^{d}}{m+1}\right)\left[\int_{\mathbb{R}^{d}} \exp \left(\frac{-\varepsilon \lambda \kappa_{d}\left\|x-y_{0}\right\|^{d}}{m+1}\right) \mathrm{d} x\right]^{m} .
\end{aligned}
$$

Using (12) completes the proof.

Lemma 4. For any $a>0$,

$$
\int_{\mathbb{R}^{d}} \int_{A} \mathrm{e}^{-a \lambda\|y-x\|^{d}} \mathrm{~d} y \mathrm{~d} x=\frac{\kappa_{d} \operatorname{Vol}(A)}{a \lambda}
$$

and

$$
\int_{\mathbb{R}^{d} \backslash A} \int_{A} \mathrm{e}^{-a \lambda\|y-x\|^{d}} \mathrm{~d} y \mathrm{~d} x \leq C \frac{\operatorname{Per}(A)}{\lambda^{1+1 / d}} \text { as } \lambda \rightarrow \infty .
$$

Proof. The first equation follows from (10) after replacing $\lambda$ by $\lambda^{\prime} a / \kappa_{d}$. The second estimate follows from (11) after replacing $\lambda$ by $\lambda^{\prime} a / \kappa_{d}$ and then applying Theorem 1.

Introduce the notation $B_{r}^{x}$ for the closed ball with centre $x \in \mathbb{R}^{d}$ and radius $r>0$ in the Euclidean metric.

Lemma 5. Let $x_{1}, x_{2}, y_{1}, y_{2} \in \mathbb{R}^{d}$. If $B_{\left\|x_{1}-y_{1}\right\|}^{x_{1}} \cap B_{\left\|x_{2}-y_{2}\right\|}^{x_{2}} \neq \varnothing$ then

$$
\mathrm{P}\left(B_{\left\|x_{1}-y_{1}\right\|}^{x_{1}} \cap \eta=\varnothing, B_{\left\|x_{2}-y_{2}\right\|}^{x_{2}} \cap \eta=\varnothing\right) \leq 2 \exp \left(-\frac{\lambda \kappa_{d}}{2^{2 d+1}}\left(\left\|x_{1}-y_{2}\right\|^{d}+\left\|x_{2}-y_{1}\right\|^{d}\right)\right) .
$$

Proof. Since $B_{\left\|x_{1}-y_{1}\right\|}^{x_{1}} \cap B_{\left\|x_{2}-y_{2}\right\|}^{x_{2}} \neq \varnothing$, it follows from the triangle inequality that

$$
\frac{\left\|x_{1}-y_{2}\right\|}{4}, \frac{\left\|x_{2}-y_{1}\right\|}{4} \leq \max \left(\left\|x_{1}-y_{1}\right\|,\left\|x_{2}-y_{2}\right\|\right) \text {. }
$$

Therefore, by Lemma 2 and the stationarity of $\eta$, we have

$$
\begin{aligned}
& \mathrm{P}\left(B_{\left\|x_{1}-y_{1}\right\|}^{x_{1}} \cap \eta=\varnothing, B_{\left\|x_{2}-y_{2}\right\|}^{x_{2}} \cap \eta=\varnothing\right) \\
& \leq \mathrm{P}\left(B_{\left\|x_{1}-y_{2}\right\| / 4}^{x_{1}} \cap \eta=\varnothing, B_{\left\|x_{2}-y_{1}\right\| / 4}^{x_{1}} \cap \eta=\varnothing\right. \\
& \left.\quad \text { or } B_{\left\|x_{1}-y_{2}\right\| / 4}^{x_{2}} \cap \eta=\varnothing, B_{\left\|x_{2}-y_{1}\right\| / 4}^{x_{2}} \cap \eta=\varnothing\right) \\
& \leq \sum_{i=1}^{2} \mathrm{P}\left(B_{\left\|x_{1}-y_{2}\right\| / 4}^{x_{i}} \cap \eta=\varnothing, B_{\left\|x_{2}-y_{1}\right\| / 4}^{x_{i}} \cap \eta=\varnothing\right) \\
& \leq \sum_{i=1}^{2}\left(\mathrm{P}\left(B_{\left\|x_{1}-y_{2}\right\| / 4}^{x_{i}} \cap \eta=\varnothing\right) \mathrm{P}\left(B_{\left\|x_{2}-y_{1}\right\| / 4}^{x_{i}} \cap \eta=\varnothing\right)\right)^{1 / 2} \\
& =2 \exp \left(-\frac{\lambda \kappa_{d}}{2^{2 d+1}}\left(\left\|x_{1}-y_{2}\right\|^{d}+\left\|x_{2}-y_{1}\right\|^{d}\right)\right) .
\end{aligned}
$$


Lemma 6. For any $x_{1}, y_{1}, \ldots, x_{n}, y_{n} \in \mathbb{R}^{d}$, it holds that

$$
\begin{aligned}
\mathrm{P}\left(B_{\left\|x_{r}-y_{r}\right\|}^{x_{r}} \cap \eta=\varnothing, r=1, \ldots, n\right) \\
\leq \exp \left(-\lambda \kappa_{d} \sum_{r=1}^{n}\left\|x_{r}-y_{r}\right\|^{d}\right) \\
+2 \sum_{s<t} \exp \left(-\frac{\lambda \kappa_{d}}{n+1} \sum_{r=1}^{n}\left\|x_{r}-y_{r}\right\|^{d}\right) \\
\quad \times \exp \left(-\frac{\lambda \kappa_{d}}{2^{2 d+1}(n+1)}\left(\left\|x_{s}-y_{t}\right\|^{d}+\left\|x_{t}-y_{s}\right\|^{d}\right)\right) .
\end{aligned}
$$

Proof. If the balls $B_{\left\|x_{r}-y_{r}\right\|}^{x_{r}}, r=1, \ldots, n$, are pairwise disjoint then we obviously have

$$
\mathrm{P}\left(B_{\left\|x_{r}-y_{r}\right\|}^{x_{r}} \cap \eta=\varnothing, r=1, \ldots, n\right)=\exp \left(-\lambda \kappa_{d} \sum_{r=1}^{n}\left\|x_{r}-y_{r}\right\|^{d}\right) .
$$

Suppose that, for some indices $s \neq t$, it holds that $B_{\left\|x_{s}-y_{s}\right\|}^{x_{s}} \cap B_{\left\|x_{t}-y_{t}\right\|}^{x_{t}} \neq \varnothing$. Applying Lemma 2, we obtain

$$
\begin{aligned}
& \mathrm{P}\left(B_{\left\|x_{r}-y_{r}\right\|}^{x_{r}} \cap \eta=\varnothing, r=1, \ldots, n\right) \\
& \quad \leq\left(\mathrm{P}\left(B_{\left\|x_{s}-y_{s}\right\|}^{x_{s}} \cap \eta=\varnothing, B_{\left\|x_{t}-y_{t}\right\|}^{x_{t}} \cap \eta=\varnothing\right)\right)^{1 /(n+1)} \prod_{r=1}^{n}\left(\mathrm{P}\left(B_{\left\|x_{r}-y_{r}\right\|}^{x_{r}} \cap \eta=\varnothing\right)\right)^{1 /(n+1)} \\
& \quad=\exp \left(-\frac{\lambda \kappa_{d}}{n+1} \sum_{r=1}^{n}\left\|x_{r}-y_{r}\right\|^{d}\right)\left(\mathrm{P}\left(B_{\left\|x_{s}-y_{s}\right\|}^{x_{s}} \cap \eta=\varnothing, B_{\left\|x_{t}-y_{t}\right\|}^{x_{t}} \cap \eta=\varnothing\right)\right)^{1 /(n+1)} .
\end{aligned}
$$

It remains to apply Lemma 5 to complete the proof.

Proof of Theorem 2. We have

$$
\begin{aligned}
\operatorname{E~Vol}^{n}\left(A_{\eta}\right) & =\mathrm{E} \int_{\left(\mathbb{R}^{d}\right)^{n}} \mathbf{1}\left(\text { there exists }\left(y_{1}, \ldots, y_{n}\right) \in(\eta \cap A)^{n}: x_{i} \in v_{\eta}\left(y_{i}\right), i=1, \ldots n\right) \\
& \times \mathrm{d} x_{1} \cdots \mathrm{d} x_{n} \\
= & \sum_{i=1}^{n} \sum_{m_{1}+\cdots+m_{i}=n} B_{n, i, m_{1}, \ldots, m_{i}} \beta_{i, m_{1}, \ldots, m_{i}},
\end{aligned}
$$

where

$$
\begin{aligned}
& \beta_{i, m_{1}, \ldots, m_{i}}=\int_{\left(\mathbb{R}^{d}\right)^{n}} \mathrm{E} \sum_{\left(y_{1}, \ldots, y_{i}\right) \in(\eta \cap A)_{\neq}^{i}} \mathbf{1}\left(x_{1}, \ldots, x_{m_{1}} \in v_{\eta}\left(y_{1}\right), \ldots, x_{n-m_{i}+1}, \ldots, x_{n} \in v_{\eta}\left(y_{i}\right)\right) \\
& \times \mathrm{d} x_{1} \cdots \mathrm{d} x_{n}
\end{aligned}
$$

and $B_{n, i, m_{1}, \ldots, m_{i}}$ denotes the number of ways to divide the set $\{1,2, \ldots, n\}$ into $i$ subsets of size $m_{1}, \ldots, m_{i}$. It is clear that

$$
B_{n, n, 1, \ldots, 1}=1 \text {. }
$$


Fix some $i$ and $m_{1}, \ldots, m_{i}$. Using the Slivnyak-Mecke formula (9), we obtain

$$
\begin{array}{rl}
\beta_{i, m_{1}, \ldots, m_{i}}=\lambda^{i} \int_{\left(\mathbb{R}^{d}\right)^{n}} \int_{A^{i}} & \mathrm{P}\left(x_{1}, \ldots, x_{m_{1}} \in v_{\eta \cup \tilde{y}_{i}}\left(y_{1}\right), \ldots, x_{n-m_{i}+1}, \ldots, x_{n} \in v_{\eta \cup \tilde{y}_{i}}\left(y_{i}\right)\right) \\
\times \mathrm{d} y_{1} \cdots \mathrm{d} y_{i} \mathrm{~d} x_{1} \cdots \mathrm{d} x_{n},
\end{array}
$$

where $\tilde{y}_{i}=\left\{y_{1}, \ldots, y_{i}\right\}$. Taking into account the fact that $v_{\eta \cup \tilde{y}_{i}}\left(y_{r}\right) \subset v_{\eta \cup\left\{y_{r}\right\}}\left(y_{r}\right)$, and using Fubini's theorem, Lemma 2, and Lemma 3, we obtain

$$
\begin{aligned}
\beta_{i, m_{1}, \ldots, m_{i}} & \leq \lambda^{i} \int_{A^{i}} \prod_{r=1}^{i} \int_{\left(\mathbb{R}^{d}\right)^{m_{r}}}\left(\mathrm{P}\left(x_{1}, \ldots, x_{m_{r}} \in v_{\eta \cup\left\{y_{r}\right\}}\left(y_{r}\right)\right)\right)^{1 / i} \mathrm{~d} x_{1} \cdots \mathrm{d} x_{m_{r}} \mathrm{~d} y_{1} \cdots \mathrm{d} y_{i} \\
& \leq \lambda^{i} \int_{A^{i}} \prod_{r=1}^{i}\left(\frac{i m_{r}}{\lambda}\right)^{m_{r}-1} \int_{\mathbb{R}^{d}}\left(\exp \left(\frac{-\lambda \kappa_{d}\left\|x_{1}-y_{r}\right\|^{d}}{i m_{r}}\right)\right) \mathrm{d} x_{1} \mathrm{~d} y_{1} \cdots \mathrm{d} y_{i} .
\end{aligned}
$$

By (15) we obtain

$$
\beta_{i, m_{1}, \ldots, m_{i}} \leq C \operatorname{Vol}^{i}(A) \lambda^{i-\sum_{r=1}^{i} m_{r}}=C \operatorname{Vol}^{i}(A) \lambda^{i-n}
$$

The maximum order of $\lambda$ is achieved for $i=n$, which together with (17) and (18) implies that

$$
\begin{aligned}
\operatorname{E~Vol}^{n}\left(A_{\eta}\right) \leq & \lambda^{n} \int_{\left(\mathbb{R}^{d}\right)^{n}} \int_{A^{n}} \mathrm{P}\left(x_{r} \in v_{\eta \cup \tilde{y}_{n}}\left(y_{r}\right), r=1, \ldots, n\right) \mathrm{d} y_{1} \cdots \mathrm{d} y_{n} \mathrm{~d} x_{1} \cdots \mathrm{d} x_{n} \\
& +C(\operatorname{Vol}(A))^{n-1} \lambda^{-1} \text { as } \lambda \rightarrow \infty
\end{aligned}
$$

It is clear that

$$
\mathrm{P}\left(x_{r} \in v_{\eta \cup \tilde{y}_{n}}\left(y_{r}\right), r=1, \ldots, n\right) \leq \mathrm{P}\left(B_{\left\|x_{r}-y_{r}\right\|}^{x_{r}} \cap \eta=\varnothing, r=1, \ldots, n\right) .
$$

Therefore, by Lemma 6 ,

$$
\mathrm{E} \mathrm{Vol}^{n}\left(A_{\eta}\right) \leq v_{n}+2 \sum_{s<t} v_{n, s, t}+C(\operatorname{Vol}(A))^{n-1} \lambda^{-1} \quad \text { as } \lambda \rightarrow \infty,
$$

where

$$
v_{n}=\lambda^{n} \int_{\left(\mathbb{R}^{d}\right)^{n}} \int_{A^{n}} \exp \left(-\lambda \kappa_{d} \sum_{r=1}^{n}\left\|x_{r}-y_{r}\right\|^{d}\right) \mathrm{d} y_{1} \cdots \mathrm{d} y_{n} \mathrm{~d} x_{1} \cdots \mathrm{d} x_{n}
$$

and

$$
\begin{aligned}
v_{n, s, t}=\lambda^{n} \int_{\left(\mathbb{R}^{d}\right)^{n}} \int_{A^{n}} & \exp \left(-\frac{\lambda \kappa_{d}}{n+1} \sum_{r=1}^{n}\left\|x_{r}-y_{r}\right\|^{d}\right) \\
& \times \exp \left(-\frac{\lambda \kappa_{d}}{2^{2 d+1}(n+1)}\left(\left\|x_{s}-y_{t}\right\|^{d}+\left\|x_{t}-y_{s}\right\|^{d}\right)\right) \\
& \times \mathrm{d} y_{1} \cdots \mathrm{d} y_{n} \mathrm{~d} x_{1} \cdots \mathrm{d} x_{n} .
\end{aligned}
$$

By (10),

$$
v_{n}=\operatorname{Vol}^{n}(A)
$$


Let us estimate $v_{n, s, t}$. Using Fubini's theorem, it follows from (15) that

$$
\begin{aligned}
& v_{n, s, t} \leq C \operatorname{Vol}^{n-2}(A) \lambda^{2} \int_{\mathbb{R}^{d}} \int_{\mathbb{R}^{d}} \int_{A} \int_{A} \exp \left(-\frac{\lambda \kappa_{d}}{n+1}\left(\left\|x_{s}-y_{s}\right\|^{d}+\left\|x_{t}-y_{t}\right\|^{d}\right)\right) \\
& \times \exp \left(-\frac{\lambda \kappa_{d}}{2^{2 d+1}(n+1)}\left(\left\|x_{s}-y_{t}\right\|^{d}+\left\|x_{t}-y_{s}\right\|^{d}\right)\right) \\
& \times \mathrm{d} y_{t} \mathrm{~d} y_{s} \mathrm{~d} x_{t} \mathrm{~d} x_{s} \\
& \leq C \operatorname{Vol}^{n-2}(A) \lambda^{2} \int_{\mathbb{R}^{d}} \int_{A} \exp \left(-\frac{\lambda \kappa_{d}}{n+1}\left(\left\|x_{s}-y_{s}\right\|^{d}\right)\right) \\
& \times \int_{\mathbb{R}^{d}} \int_{\mathbb{R}^{d}} \exp \left(-\frac{\lambda \kappa_{d}}{2^{2 d+1}(n+1)}\left(\left\|x_{s}-y_{t}\right\|^{d}+\left\|x_{t}-y_{s}\right\|^{d}\right)\right) \\
& \times \mathrm{d} y_{t} \mathrm{~d} x_{t} \mathrm{~d} y_{s} \mathrm{~d} x_{s} .
\end{aligned}
$$

Furthermore, by (12),

$$
v_{n, s, t} \leq C \operatorname{Vol}^{n-2}(A) \int_{\mathbb{R}^{d}} \int_{A} \exp \left(-\frac{\lambda \kappa_{d}}{n+1}\left\|x_{s}-y_{s}\right\|^{d}\right) \mathrm{d} y_{s} \mathrm{~d} x_{s},
$$

and applying (15) again, we obtain

$$
v_{n, s, t} \leq C \operatorname{Vol}^{n-1}(A) \lambda^{-1} .
$$

Combining this with estimate (19) and (20), we obtain

$$
\mathrm{EVol}^{n}\left(A_{\eta}\right) \leq \operatorname{Vol}^{n}(A)+C \operatorname{Vol}^{n-1}(A) \lambda^{-1} \text { as } \lambda \rightarrow \infty .
$$

The application of Lyapunov's inequality

$$
\mathrm{EVol}^{n}\left(A_{\eta}\right) \geq\left(\operatorname{EVol}\left(A_{\eta}\right)\right)^{n}=\operatorname{Vol}^{n}(A)
$$

completes the proof.

Proof of Theorem 3. We have

$$
\mathrm{EVol}^{n}\left(A \Delta A_{\eta}\right)=\mathrm{E}\left(\operatorname{Vol}\left(A \backslash A_{\eta}\right)+\operatorname{Vol}\left(A_{\eta} \backslash A\right)\right)^{n}=\sum_{k=0}^{n}\left(\begin{array}{l}
n \\
k
\end{array}\right) u_{k},
$$

where

$$
u_{k}=\mathrm{E} \int_{A^{n-k}} \int_{\left(\mathbb{R}^{d} \backslash A\right)^{k}} \mathbf{1}\left(x_{1}, \ldots, x_{k} \in A_{\eta}, x_{k+1}, \ldots, x_{n} \notin A_{\eta}\right) \mathrm{d} x_{1} \cdots \mathrm{d} x_{n} .
$$

Fix some $k$. We have

$$
\begin{aligned}
u_{k} & =\mathrm{E} \int_{A^{n-k}} \int_{\left(\mathbb{R}^{d} \backslash A\right)^{k}} \mathbf{1}\left(\text { there exist }\left(y_{1}, \ldots, y_{k}\right) \in(\eta \cap A)^{k},\right. \\
& =\sum_{i=1}^{k} \sum_{j=1}^{n-k} \sum_{\left.\left.y_{k+1}, \ldots, y_{n}\right) \in(\eta \backslash A)^{n-k}: x_{i} \in v_{\eta}\left(y_{i}\right), i=1, \ldots, n\right) \mathrm{d} x_{1} \cdots \mathrm{d} x_{n}} \sum_{m_{1}+\cdots+m_{i}=k l_{1}+\cdots+l_{j}=n-k} B_{k, i, m_{1}, \ldots, m_{i}} B_{n-k, j, l_{1}, \ldots, l_{j}} \beta_{i, j, m_{1}, \ldots, m_{i}, l_{1}, \ldots, l_{j},},
\end{aligned}
$$


where

$$
\begin{gathered}
\beta_{i, j, m_{1}, \ldots, m_{i}, l_{1}, \ldots, l_{j}} \int_{A^{n-k}} \int_{\left(\mathbb{R}^{d} \backslash A\right)^{k}} \mathrm{E} \sum_{\left(y_{1}, \ldots, y_{i}\right) \in(\eta \cap A)_{\neq}^{i} \neq\left(y_{i+1}, \ldots, y_{i+j}\right) \in(\eta \backslash A)_{\neq}^{j}} \mathbf{1}\left(x_{1}, \ldots, x_{m_{1}} \in v_{\eta}\left(y_{1}\right), \ldots,\right. \\
\left.x_{n-l_{j}+1}, \ldots, x_{n} \in v_{\eta}\left(y_{i+j}\right)\right) \\
\times \mathrm{d} x_{1} \cdots \mathrm{d} x_{n}
\end{gathered}
$$

and $B_{k, i, m_{1}, \ldots, m_{i}}, B_{n-k, j, l_{1}, \ldots, l_{j}}$ are the same combinatorial coefficients as in the proof of Theorem 2.

Fix some $i, j$, and $m_{1}, \ldots, m_{i}, l_{1}, \ldots, l_{j}$. Using the Slivnyak-Mecke formula (9) twice, we obtain

$$
\begin{aligned}
& \beta_{i, j, m_{1}, \ldots, m_{i}, l_{1}, \ldots, l_{j}} \int_{A^{n-k}} \int_{\left(\mathbb{R}^{d} \backslash A\right)^{k}} \int_{\left(\mathbb{R}^{d} \backslash A\right)^{j}} \int_{A^{i}} \mathrm{P}\left(x_{1}, \ldots, x_{m_{1}} \in v_{\eta \cup \tilde{y}_{i+j}}\left(y_{1}\right), \ldots,\right. \\
& \left.x_{n-l_{j}+1}, \ldots, x_{n} \in v_{\eta \cup \tilde{y}_{i+j}}\left(y_{i+j}\right)\right) \\
& \times \mathrm{d} y_{1} \cdots \mathrm{d} y_{i+j} \mathrm{~d} x_{1} \cdots \mathrm{d} x_{n},
\end{aligned}
$$

where $\tilde{y}_{i+j}=\left\{y_{1}, \ldots, y_{i+j}\right\}$. By Fubini's theorem and Lemma 2,

$$
\begin{aligned}
& \beta_{i, j, m_{1}, \ldots, m_{i}, l_{1}, \ldots, l_{j}} \\
& \leq \lambda^{i+j} \int_{\left(\mathbb{R}^{d} \backslash A\right)^{j}} \int_{A^{i}} \prod_{r=1}^{i} \int_{\left(\mathbb{R}^{d} \backslash A\right)^{m_{r}}}\left(\mathrm{P}\left(x_{1}, \ldots, x_{m_{r}} \in v_{\eta \cup\left\{y_{r}\right\}}\left(y_{r}\right)\right)\right)^{1 /(i+j)} \mathrm{d} x_{1} \cdots \mathrm{d} x_{m_{r}} \\
& \times \prod_{r=1}^{j} \int_{A^{l_{r}}}\left(\mathrm{P}\left(x_{1}, \ldots, x_{l_{r}} \in v_{\eta \cup\left\{y_{i+r}\right\}}\left(y_{i+r}\right)\right)\right)^{1 /(i+j)} \mathrm{d} x_{1} \cdots \mathrm{d} x_{l_{r}} \\
& \times \mathrm{d} y_{1} \cdots \mathrm{d} y_{i+j} .
\end{aligned}
$$

Using Lemma 3 and (16), we obtain, asymptotically as $\lambda \rightarrow \infty$,

$$
\begin{aligned}
& \beta_{i, j, m_{1}, \ldots, m_{i}, l_{1}, \ldots, l_{j}} \leq C \operatorname{Per}(A)^{i+j} \lambda^{i+j+\sum_{r=1}^{i}\left(-m_{r}-1 / d\right)+\sum_{r=1}^{j}\left(-l_{r}-1 / d\right)} \\
&=C \operatorname{Per}(A)^{i+j} \lambda^{-n+i+j-(i+j) / d} .
\end{aligned}
$$

The maximum order of $\lambda$ is achieved for $i=k, j=n-k$, and the next term of maximum order is achieved for $i+j=n-1$, which together with (22) and (18) implies that, asymptotically as $\lambda \rightarrow \infty$,

$$
\begin{aligned}
u_{k} \leq & \lambda^{n} \int_{A^{n-k}} \int_{\left(\mathbb{R}^{d} \backslash A\right)^{k}} \int_{\left(\mathbb{R}^{d} \backslash A\right)^{n-k}} \int_{A^{k}} \mathrm{P}\left(x_{r} \in v_{\eta \cup \tilde{y}_{n}}\left(y_{r}\right), r=1, \ldots, n\right) \\
& \times \mathrm{d} y_{1} \cdots \mathrm{d} y_{n} \mathrm{~d} x_{1} \cdots \mathrm{d} x_{n} \\
& +C \operatorname{Per}(A)^{n-1} \lambda^{-1-(n-1) / d .}
\end{aligned}
$$


It is clear that

$$
\mathrm{P}\left(x_{r} \in v_{\eta \cup \tilde{y}_{n}}\left(y_{r}\right), r=1, \ldots, n\right) \leq \mathrm{P}\left(B_{\left\|x_{r}-y_{r}\right\|}^{x_{r}} \cap \eta=\varnothing, r=1, \ldots, n\right) .
$$

Therefore, by Lemma 6 , asymptotically as $\lambda \rightarrow \infty$,

$$
u_{k} \leq v_{k}+2 \sum_{s<t} v_{k, s, t}+C \operatorname{Per}(A)^{n-1} \lambda^{-1-(n-1) / d},
$$

where

$$
v_{k}=\lambda^{n} \int_{A^{n-k}} \int_{\left(\mathbb{R}^{d} \backslash A\right)^{k}} \int_{\left(\mathbb{R}^{d} \backslash A\right)^{n-k}} \int_{A^{k}} \exp \left(-\lambda \kappa_{d} \sum_{r=1}^{n}\left\|x_{r}-y_{r}\right\|^{d}\right) \mathrm{d} y_{1} \cdots \mathrm{d} y_{n} \mathrm{~d} x_{1} \cdots \mathrm{d} x_{n}
$$

and

$$
\begin{aligned}
v_{k, s, t}=\lambda^{n} \int_{A^{n-k}} \int_{\left(\mathbb{R}^{d} \backslash A\right)^{k}} \int_{\left(\mathbb{R}^{d} \backslash A\right)^{n-k}} \int_{A^{k}} & \exp \left(-\frac{\lambda \kappa_{d}}{n+1} \sum_{r=1}^{n}\left\|x_{r}-y_{r}\right\|^{d}\right) \\
& \times \exp \left(-\frac{\lambda \kappa_{d}}{2^{2 d+1}(n+1)}\left(\left\|x_{s}-y_{t}\right\|^{d}+\left\|x_{t}-y_{s}\right\|^{d}\right)\right) \\
& \times \mathrm{d} y_{1} \cdots \mathrm{d} y_{n} \mathrm{~d} x_{1} \cdots \mathrm{d} x_{n} .
\end{aligned}
$$

By identity (11),

$$
v_{k}=2^{-n}\left(\mathrm{E} \operatorname{Vol}\left(A \Delta A_{\eta}\right)\right)^{n} .
$$

Let us estimate $v_{k, s, t}$. For instance, we assume that $s \leq k$ and $t \geq k+1$ (other cases are treated in the same way). In the same way as in the proof of Theorem 2, we obtain, by inequality (16),

$$
\begin{aligned}
& v_{k, s, t} \leq C \operatorname{Per}(A)^{n-2} \lambda^{2-(n-2) / d} \\
& \times \int_{\mathbb{R}^{d}} \int_{\mathbb{R}^{d} \backslash A} \int_{\mathbb{R}^{d}} \int_{A} \exp \left(-\frac{\lambda \kappa_{d}}{n+1}\left(\left\|x_{s}-y_{s}\right\|^{d}\right)\right) \\
& \times \exp \left(-\frac{\lambda \kappa_{d}}{2^{2 d+1}(n+1)}\left(\left\|x_{s}-y_{t}\right\|^{d}+\left\|x_{t}-y_{s}\right\|^{d}\right)\right) \\
& \times \mathrm{d} y_{s} \mathrm{~d} y_{t} \mathrm{~d} x_{s} \mathrm{~d} x_{t}
\end{aligned}
$$

as $\lambda \rightarrow \infty$. Furthermore, by (12),

$$
v_{k, s, t} \leq C \operatorname{Per}(A)^{n-2} \lambda^{-(n-2) / d} \int_{\mathbb{R}^{d} \backslash A} \int_{A} \exp \left(-\frac{\lambda \kappa_{d}}{n+1}\left\|x_{s}-y_{s}\right\|^{d}\right) \mathrm{d} y_{s} \mathrm{~d} x_{s} \quad \text { as } \lambda \rightarrow \infty,
$$

and applying (16) again, we obtain

$$
v_{k, s, t} \leq C \operatorname{Per}(A)^{n-1} \lambda^{-1-(n-1) / d} \quad \text { as } \lambda \rightarrow \infty .
$$


Combining this with (23) and (24), we obtain

$$
u_{k} \leq 2^{-n}\left(\operatorname{EVol}\left(A \Delta A_{\eta}\right)\right)^{n}+C \operatorname{Per}(A)^{n-1} \lambda^{-1-(n-1) / d} \quad \text { as } \lambda \rightarrow \infty .
$$

Inserting this into (21) we obtain

$$
\mathrm{E} \mathrm{Vol}^{n}\left(A \Delta A_{\eta}\right) \leq\left(\mathrm{EVol}\left(A \Delta A_{\eta}\right)\right)^{n}+C \operatorname{Per}(A)^{n-1} \lambda^{-1-(n-1) / d} \quad \text { as } \lambda \rightarrow \infty .
$$

The application of Lyapunov's inequality

$$
\mathrm{E} \mathrm{Vol}^{n}\left(A \Delta A_{\eta}\right) \geq\left(\operatorname{EVol}\left(A \Delta A_{\eta}\right)\right)^{n}
$$

completes the proof.

Proof of Corollary 2. As mentioned above, the second inequality immediately follows from Theorem 3. To prove the first inequality, let us again combine (21) and (25) now for $n=2$ and $k=0,1,2$. We obtain, for sufficiently large $\lambda$,

$$
\mathrm{E} \mathrm{Vol}^{2}\left(A \backslash A_{\eta}\right)+\mathrm{E} \mathrm{Vol}^{2}\left(A_{\eta} \backslash A\right) \leq \frac{1}{2}\left(\operatorname{EVol}\left(A \Delta A_{\eta}\right)\right)^{2}+2 C \operatorname{Per}(A) \lambda^{-1-1 / d}
$$

and

$$
2 \mathrm{E}\left(\operatorname{Vol}\left(A \backslash A_{\eta}\right) \operatorname{Vol}\left(A_{\eta} \backslash A\right)\right) \leq \frac{1}{2}\left(\operatorname{EVol}\left(A \Delta A_{\eta}\right)\right)^{2}+2 C \operatorname{Per}(A) \lambda^{-1-1 / d} .
$$

Combining this with Lyapunov's inequality

$$
\mathrm{E} \mathrm{Vol}^{2}\left(A \backslash A_{\eta}\right)+\mathrm{E} \mathrm{Vol}^{2}\left(A_{\eta} \backslash A\right)+2 \mathrm{E}\left(\operatorname{Vol}\left(A \backslash A_{\eta}\right) \operatorname{Vol}\left(A_{\eta} \backslash A\right)\right) \geq\left(\mathrm{EVol}\left(A \Delta A_{\eta}\right)\right)^{2},
$$

we obtain, for sufficiently large $\lambda$,

$$
\mathrm{E} \mathrm{Vol}^{2}\left(A \backslash A_{\eta}\right)+\mathrm{E} \mathrm{Vol}^{2}\left(A_{\eta} \backslash A\right)-2 \mathrm{E}\left(\operatorname{Vol}\left(A \backslash A_{\eta}\right) \operatorname{Vol}\left(A_{\eta} \backslash A\right)\right) \leq 4 C \operatorname{Per}(A) \lambda^{-1-1 / d},
$$

which together with (13) completes the proof.

\section{References}

[1] Ambrosio, L., Colesanti, A. And Villa, E. (2008). Outer Minkowski content for some classes of closed sets. Math. Ann. 342, 727-748.

[2] Ambrosio, L., Fusco, N. and Pallara, D. (2000). Functions of Bounded Variation and Free Discontinuity Problems. Oxford University Press, New York.

[3] Averkov, G. ANd Bianchi, G. (2009). Confirmation of Matheron's conjecture on the covariogram of a planar convex body. J. Europ. Math. Soc. 11, 1187-1202.

[4] Billingsley, P. (1979). Probability and Measure. John Wiley, New York.

[5] Einmahl, J. H. J. and Khmaladze, E. V. (2001). The two-sample problem in $\mathbb{R}^{m}$ and measure-valued martingales. In State of the Art in Probability and Statistics (Leiden, 1999; IMS Lecture Notes Monogr. Ser. 36), Institute of Mathematical Statistics, Beachwood, OH, pp. 434-463.

[6] Galerne, B. (2011). Computation of the perimeter of measurable sets via their covariogram. Applications to random sets. Image Anal. Stereol. 30, 39-51.

[7] Heveling, M. and Reitzner, M. (2009). Poisson-Voronoi approximation. Ann. Appl. Prob. 19, 719-736.

[8] Khmaladze, E. and Toronjadze, N. (2001). On the almost sure coverage property of Voronoi tessellation: the $\mathbb{R}^{1}$ case. Adv. Appl. Prob. 33, 756-764.

[9] Last, G. and Penrose, M. D. (2011). Poisson process Fock space representation, chaos expansion and covariance inequalities. Prob. Theory Relat. Fields 150, 663-690.

[10] MøLler, J. (1994). Lectures on Random Voronŏ Tessellations (Lecture Notes Statist. 87). Springer, New York.

[11] Penrose, M. D. (2007). Laws of large numbers in stochastic geometry with statistical applications. Bernoulli 13, 1124-1150.

[12] SchneIder, R., AND WeIL, W. (2008). Stochastic and Integral Geometry. Springer, Berlin. 
[13] Schulte, M. (2012). A central limit theorem for the Poisson-Voronoi approximation. Adv. Appl. Math. 49, 285-306.

[14] Stoyan, D., Kendall, W. S. And Mecke, J. (1995). Stochastic Geometry and Its Applications, 2nd edn. John Wiley, Chichester.

[15] Wu, L. (2000). A new modified logarithmic Sobolev inequality for Poisson point processes and several applications. Prob. Theory Relat. Fields 118, 427-438. 